\title{
Reflexive Polyhedra and their Applications in String and F-theory
}

\author{
Harald Skarke \\ Institut für Physik, Humboldt-Universität zu Berlin, \\ Invalidenstraße 110, D-10115 Berlin, Germany \\ skarkẹpysik. hu-berlin. de
}

ABstract: This is an informal introduction to the concept of reflexive polyhedra and some of their most important applications in perturbative and non-perturbative string physics. Following the historical development, topics like mirror symmetry, gauged linear sigma models, and the geometrical structures relevant to string and F-theory dualities are discussed. Finally some recent developments concerning the classification of reflexive polyhedra are mentioned.

\section{Perturbative string theory and re- flexive polyhedra}

Originally the introduction of reflexive polyhedra was motivated by mirror symmetry: A superstring compactification whose target space is a Calabi-Yau threefold leads, on the string world sheet, to an $N=2$ superconformal field theory with central charge $c=9$. The $N=2$ superalgebra of this world sheet theory decomposes into a right and a left moving part, each of which has a $U(1)$ R-symmetry. A change in the relative signs of the left/right $U(1)$ charges is a trivial operation from the point of view of world sheet dynamics, but it radically changes the spacetime interpretation of the model: Assuming that the model has a geometrical interpretation before and after the sign flip, this implies that we have passed from a Calabi-Yau manifold to a different Calabi-Yau manifold in such a way that the Hodge numbers $h_{11}$ and $h_{12}$ are interchanged. The conjecture that most Calabi-Yau manifolds have mirrors of this kind was supported by the first explicit constructions of large classes of such manifolds as hypersurfaces in weighted projective spaces [i]1]: It was found that for most of the resulting Hodge pairs the mirror pair could also be found. The completion of the classification of Calabi-Yau hypersurfaces in weighted projec- tive spaces of the mirrors could be found within the same class. The resolution to this problem was provided by the introduction of Calabi-Yau manifolds that are constructed as hypersurfaces in toric varieties with the help of so-called reflexive polyhedra [i] vided not only the missing mirrors but also many completely new models.

Reflexive polyhedra are defined with respect to a dual pair of lattices $M \simeq \mathbb{Z}^{n}$ and $N \simeq \mathbb{Z}^{n}$ and the underlying real vector spaces $M_{\mathbb{R}} \simeq \mathbb{R}^{n}$ and $N_{\mathbb{R}} \simeq \mathbb{R}^{n}$. They are polytopes in these real vector spaces with the origin in their respective interiors. For any such polytope $\Delta \subset M_{\mathbb{R}}$ one can define the dual polytope as

$$
\Delta^{*}:=\left\{v \in N_{\mathbb{R}}:\langle v, w\rangle \geq-1 \forall w \in \Delta\right\} .
$$

A lattice polyhedron $\Delta$ is a polyhedron in $M_{\mathbb{R}}$ with vertices in $M$, and a reflexive polyhedron is a lattice polyhedron $\Delta$ with $\mathbf{0}$ in its interior such that $\Delta^{*}$ is also a lattice polyhedron.

From such polytopes, complex manifolds can be constructed in the following simple way: One draws rays $v_{i}$ through the vertices (or, more generally, through lattice points) of $\Delta^{*}$ and introduces a homogeneous coordinate $x_{i}$ for every ray $v_{i}$ in a way similar to the construction of projective space. Then one must find a complete set 

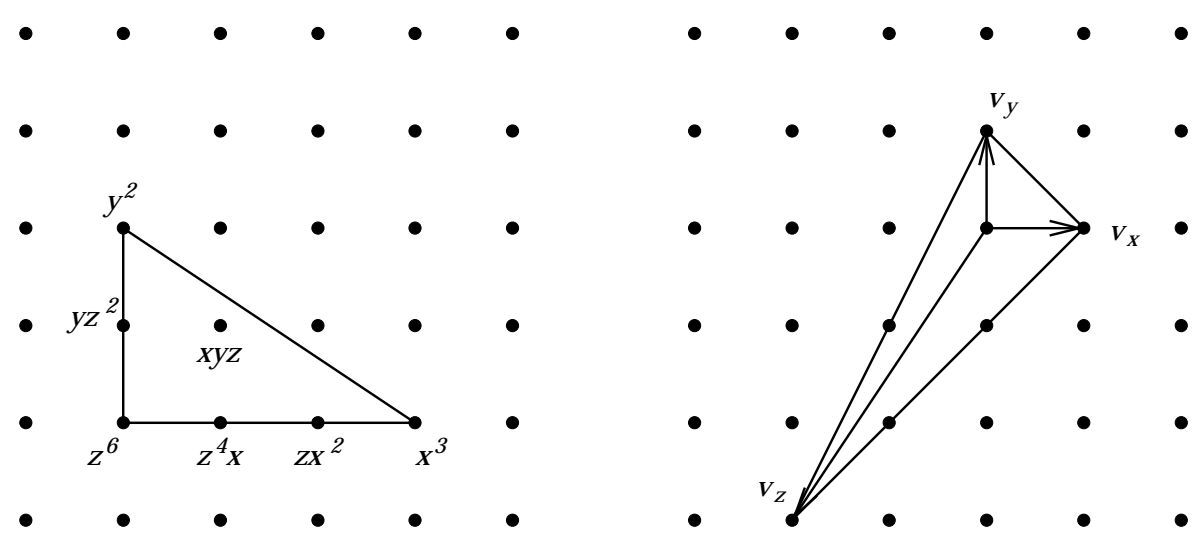

Figure 1: $\Delta$ and $\Delta^{*}$ for a weighted projective space

of linear relations of the type $\sum q_{i} v_{i}=0$ among the lattice vectors defining these rays, and for every such linear relation one introduces a multiplicative equivalence relation $x_{i} \simeq \lambda^{q_{i}} x_{i}$ among the homogeneous coordinates. This is most easily visualized with an example: For the reflexive pair of fig. 1 , the rays corresponding to the vertices of $\Delta^{*}$ fulfil $2 v_{x}+3 v_{y}+v_{z}=0$, so the space described in this way is just the weighted projective space defined as the set of equivalence classes in $\mathbb{C}^{3} \backslash\{0\}$ with respect to the relations $(x, y, z) \simeq\left(\lambda^{2} x, \lambda^{3} y, \lambda z\right)$. It is not hard to see that this space suffers from singularities at the points $y=z=0$ and $x=z=0$. In algebraic geometry there is a procedure for turning such a singular space into a smooth one, referred to as 'blowing up' singularities. In the toric setup, it is very easy to perform this operation: It simply corresponds to adding extra rays to the toric diagram; in the present example these are just the rays through the lattice points interior to edges of $\Delta^{*}$.

In order to define hypersurfaces in toric varieties, it is necessary to have equations that are compatible with the equivalence relations among the toric coordinates. For the case of Calabi-Yau hypersurfaces, these homogeneous equations are particularly simple. In terms of rays $v_{i}$, corresponding coordinates $x_{i}$ and lattice points $w$ of $\Delta$, a Calabi-Yau hypersurface is determined by

$$
P\left(x_{1}, \ldots, x_{k}\right)=\sum_{w \in \Delta \cap M} a_{w} \prod_{i=1}^{k} x_{i}^{\left\langle v_{i}, w\right\rangle+1}=0,
$$

i.e. every lattice point of $\Delta$ determines a mono- mial in $P$. For our example, which leads to a one dimensional Calabi-Yau hypersurface which is nothing but an elliptic curve, the corresponding monomials are indicated in fig.1. In the context of polyhedra mirror symmetry manifests itself as the exchange of $(M, \Delta)$ and $\left(N, \Delta^{*}\right)$.

Almost immediately after its introduction this construction turned out to be important for further applications in the context of string theory: Witten's gauged linear sigma models $[\bar{n} \bar{n} \mid]$ are $d=2$ theories with $N=2$ supersymmetry. In such a model a chiral superfield is introduced for every toric coordinate and $U(1)$ gauge fields implement the compact parts of the multiplicative relations. The superpotential is just the previously introduced homogeneous polynomial multiplied with an auxiliary field. At the fixed points of the renormalization group flow, such a model is believed to possess superconformal symmetry. Within a certain range of the coupling constants the following scenario takes place: The equations of motion constrain the superfields in such a way that the noncompact part of the relations is also fixed and the homogeneous polynomial is forced to vanish, leading effectively to a CalabiYau sigma model. For other values, one gets a Landau-Ginzburg model or some hybrid. As

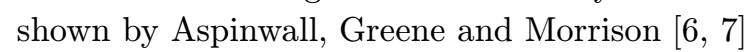
this construction implies that the corresponding string physics allows for physically smooth topology changing transitions. 


\section{String dualities and reflexive poly- hedra}

One of the main new insights of the second string revolution is that there appear to be non-perturbative dualities between seemingly different theories. Examples of this type are the dualities between heterotic string theory compactified on $K 3 \times T^{2}$ and type IIA on a $K 3$ fibered Calabi-Yau threefold and between heterotic string theory compactified on Calabi-Yau $n$-folds and F-theory on elliptically fibered Calabi-Yau $(n+1)$-folds. While heterotic string theory has non-abelian gauge groups in its perturbative spectrum, the dual theories can develop them only if the compactification manifold becomes singular. Certain singularities allow for a classification that follows exactly the pattern of the classification of simply laced Lie groups, and the non-perturbatively enhanced gauge groups are just the corresponding ADE gauge groups. Thus the two most important geometrical ingredients for making these dualities work are fibration structures and singularities. As we will now see, both types of structures manifest themselves in simple ways in the context of reflexive polyhedra.

A manifold has a fibration structures if there is a projection to some other manifold called the base manifold, and if the preimage of a generic point of the base under this projection is isomorphic to another manifold which is the fiber. In terms of reflexive polytopes, the fiber corresponds to a reflexive subpolytope $\Delta_{\text {fiber }}^{*} \subset \Delta_{\text {total }}^{*}$ in a linear subspace $N_{\mathbb{R} \text {,fiber }} \subset N_{\mathbb{R}}$ containing $\mathbf{0}$. The base space is just the toric variety whose fan (i.e., collection of rays and some other data) is the projection of the complete fan along the subspace $N_{\mathbb{R}, \text { fiber }}$. As an example, fig. $\overline{2}$ ' shows the fan of a smooth elliptically fibered K3 surface, with the fiber determined by the triangle $v_{x} v_{y} v_{z}$. The toric diagram for the base space $\mathbb{P}^{1}$ is determined by projecting along the plane of the triangle. It is a line segment isomorphic to $v_{s} v_{t}$.

ADE singularities manifest themselves in a particularly elegant way in terms of reflexive polyhedra: As Candelas and Font observed [is]], under favourable circumstances the Dynkin diagrams of non-perturbative gauge groups can be seen in

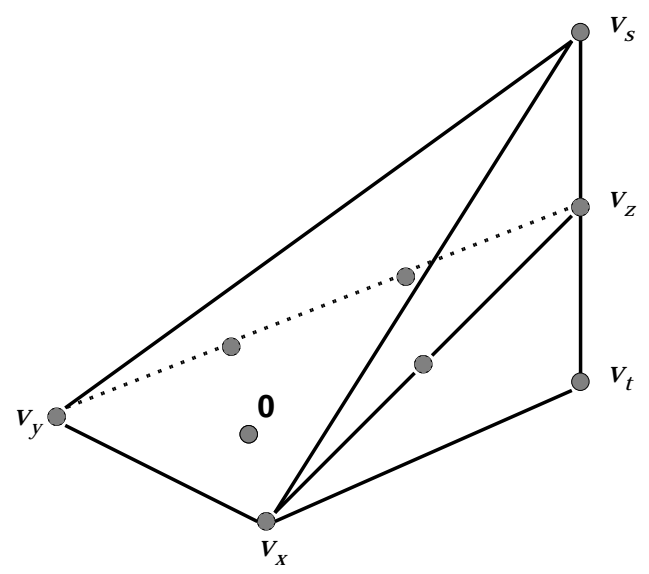

Figure 2: The polyhedron $\Delta^{*}$ corresponding to a smooth elliptically fibered K3 surface

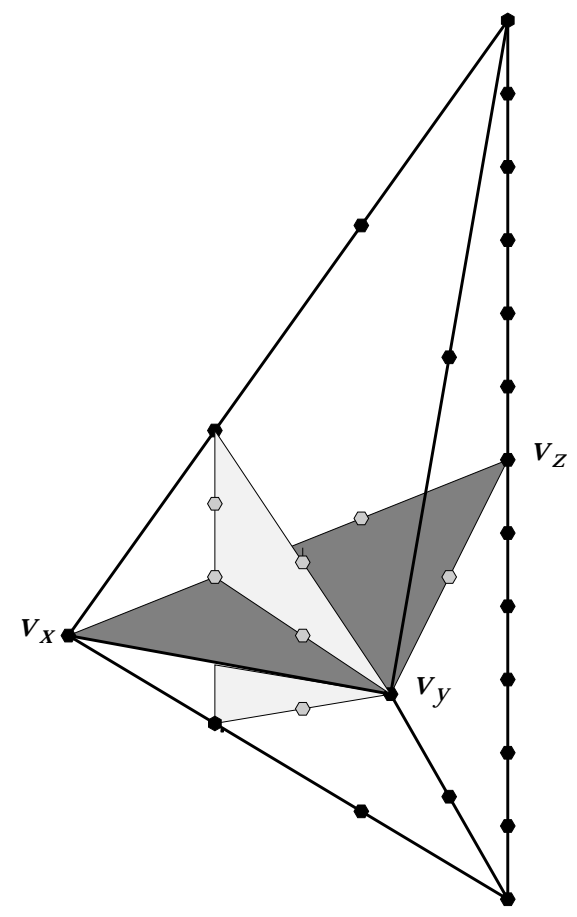

Figure 3: The polyhedron $\Delta^{*}$ corresponding to the heterotic string with unbroken gauge symmetry

the polyhedra corresponding to the blow-up of the singular space. As an example fig. ${ }^{3}$ shows the reflexive polytope that provides the F-theory dual of heterotic string theory compactified to eight dimensions with an unbroken gauge group of $E_{8} \times E_{8}$. The horizontal triangle represents the elliptic fiber, and the diagram obtained by considering the lattice points of $\Delta^{*}$ above it and the edges connecting them is nothing but the ex- 
tended Dynkin diagram of $E_{8}$ (the other $E_{8}$ is visible in the same way below the triangle). Using this polytope for constructing threefolds with the corresponding K3 manifold as a fiber, it was possible to obtain theories in six dimensions with gauge groups like $E_{8} \times\left(E_{8} \times F_{4} \times G_{2}^{2} \times A_{1}^{2}\right)^{16}[\overline{9} \cdot$. This was actually the 'record gauge group' until Aspinwall and Morrison constructed a model with the group

$S O(32) \times S p(24) \times S O(80) \times S p(48) \times S O(128)$ $\times S p(72) \times S O(176) \times S p(40) \times S p(52) \times S O(64)$, using $S O(32)$ heterotic string theory. This presented the challenge of finding the F-theory dual of the $S O(32)$ heterotic string. Remarkably, it is represented again by the polytope of fig. This time the fibration structure is determined by the vertical triangle and the lattice points to the right of it are connected by the edges into the extended Dynkin diagram of $S O(32)$.

\section{Classification of reflexive polyhe- dra}

Given all these interesting properties of reflexive polyhedra, one may certainly wonder how many of them exist in a given dimension $d$. Until 1998, this was known only for the case of $d=2$ with 16 reflexive polygons. This situation has changed with the development of a classification scheme that works (in principle) in arbitrary dimensions $\left[1 \overline{1}_{1}^{1}, \mathbf{1}_{2}\right]$. The main ideas of this scheme are as follows: Clearly it is enough to find a finite set of polyhedra that contain all reflexive polytopes as subpolytopes. By duality, $\Delta_{1} \subset \Delta_{2} \Leftrightarrow \Delta_{2}^{*} \subset \Delta_{1}^{*}$, so this task is equivalent to finding a set of polyhedra such that every reflexive polytope contains one of them. In this spirit we define a minimal polyhedron $\nabla \subset N_{\mathbb{R}}$ as a polyhedron with $\mathbf{0}$ in its interior such that the convex hull of any proper subset of vertices of $\nabla$ fails to have $\mathbf{0}$ in its interior. It can be shown that the vertices of minimal polyhedra always belong to (possibly lower dimensional) simplices with $\mathbf{0}$ in their respective interiors, and the different simplex structures can be classified. In the same way as in the first section, these simplices determine weight systems corresponding to the linear relations of the vertices. The weight systems of the simplices in a minimal polytope together form a combined weight system which uniquely specifies the linear structure of $\nabla$, but not the lattice. The additional restrictions that $\nabla$ should be a lattice polytope and that the convex hull of the intersection of $\nabla^{*}$ with $M$ should have $\mathbf{0}$ in its interior lead to finite numbers of combined weight systems. Thus all reflexive polyhedra can be obtained as subpolyhedra of the convex hulls of $\nabla^{*} \cap M$, where $\nabla$ is given by some combined weight system.

There are certain subtleties in this approach: One should take into account that the lattice is not always uniquely determined by the weights, but there are methods to deal with sublattices of the finest possible $M$ lattice which is dual to the lattice generated by the vertices of $\nabla$. Given the fact that two polytopes should be considered equivalent if they are related by lattice isomorphisms it is necessary to define normal forms of polyhedra that take this difficulty into account. Finally, in the case of four dimensional reflexive polyhedra one nearly encounters the limits of today's available computer power: Unless numerous tricks are used, there will be numerical overflows, lack of RAM and disk space, and an enormous computation time.

Nevertheless, the algorithm works and has produced the following results: In $d=3$, there are 4319 reflexive polyhedra corresponding to K3 surfaces [1길. which can be produced by a modern PC within 8 seconds. For the case of $d=4$, the programs were still running at the time this talk was given and had produced around 300 million polyhedra. At that time I estimated the total number to be around 0.5 $\pm 0.1 \mathrm{GCY}$ (GigaCalabi-Yau). This estimate was confirmed by the recent accomplishment of this project after a computation time of more than half a year on several processors: There are 473,800,776 reflexive polyhedra giving rise to 30,108 distinct pairs of Hodge numbers for Calabi-Yau threefolds [1,

\section{Acknowledgments}

The European Union TMR project ERBFMRXCT-96-0045 is not only the organizer of the conference where this talk was delivered, but has also supported my research since November 1998. Thanks! 


\section{References}

[1] P. Candelas, M. Lynker and R. Schimmrigk, Calabi-Yau manifolds in weighted $\mathbb{P}_{4}$, Nucl. Phys. B341 (1990) 383.

[2] A. Klemm, R. Schimmrigk, Landau-Ginzburg string vacua, Nucl. Phys. B411 (1994) 559, hep-th/9204060.

[3] M. Kreuzer, H. Skarke, No mirror symmetry in Landau-Ginzburg spectra!, Nucl. Phys. B388 (1992) 113, hep-th/9205004.

[4] V.V. Batyrev, Dual polyhedra and mirror symmetry for Calabi-Yau hypersurfaces in toric varieties, J. Alg. Geom. 3 (1994) 493, alg-geom/9310003:

[5] E. Witten, Phases of $N=2$ theories in two dimensions, Nucl. Phys. B403 (1993) 159, hep-th/9301042.

[6] P.S. Aspinwall, B.R. Greene, D.R. Morrison, Multiple Mirror Manifolds and Topology Change in String Theory, Phys. Lett. B303 (1993) 249, hep-th/9301043.

[7] P.S. Aspinwall, B.R. Greene, D.R. Morrison, Calabi-Yau Moduli Space, Mirror Manifolds and Spacetime Topology Change in String Theory, Nucl. Phys. B416 (1994) 414, hep-th/9309097..

[8] P. Candelas and A. Font, Duality Between the Webs of Heterotic and Type II Vacua, Nucl. Phys. B511 (1998) 295, hep-th/9603170'.

[9] P. Candelas, E. Perevalov and G. Rajesh, FTheory Duals of Nonperturbative Heterotic E8 $\times$ E8 Vacua in Six Dimensions, Nucl. Phys. $\mathbf{B 5 0 2}$ (1997) 613, hep-th/9606133'

[10] P. Candelas, H. Skarke, F-theory, SO(32) and Toric Geometry, Phys. Lett. B413 (1997) 63, hep-th/9706226.

[11] M. Kreuzer, H.Skarke, On the classification of reflexive polyhedra, Commun. Math. Phys. 185 (1997) 495, hep-th/9512204.

[12] H. Skarke, Weight systems for toric CalabiYau varieties and reflexivity of Newton polyhedra, Mod. Phys. Lett. A11 (1996) 1637, alg-geom/9603007:

[13] M. Kreuzer, H. Skarke, Classification of Reflexive Polyhedra in Three Dimensions, Adv. Theor. Math. Phys. 2 (1998) 847, "hep-th/9805190.'.

[14] M. Kreuzer, H. Skarke, Complete classification of reflexive polyhedra in four dimensions, hep-th/0002240. 